\title{
ERROR ASSESSMENT ON THE PLANNING STAGE OF NATIONAL RADON CASE-CONTROL STUDY*
}

\author{
A. Onishchenko**, A. Varaksin, I. Yarmoshenko, M. Zhukovsky
}

Institute of Industrial Ecology UB RAS, Yekaterinburg, Russia

\begin{abstract}
The possible influence of errors of radon exposure assessment on the results of planned national casecontrol study has been analyzed. It is demonstrated that, in general, the errors are classical multiplicative errors. It is demonstrated that the classical multiplicative errors of radon concentration measurement are not constant in all radon concentration ranges. At low radon concentrations, the additional influence of Poisson error on the measurement result should be considered. The modeling of the influence of errors of radon exposure assessment on slope factor of the dependence of lung cancer incidence on radon concentration in dwellings was conducted. It was shown that the expected level of radon exposure errors can reduce the observed value of slope coefficient at least twice in comparison with the original value for error-free study. The correction of the results of linear assessment of exposure-effect slope coefficient under the influence of measurement errors was realized by regression calibration technique and SIMEX extrapolation method. Regression calibration method gives the best results in restoring the original unbiased value of exposure-effect slope coefficient. The SIMEX method also allows the obtainment of the good assessment of expected value of the slope of exposure-effect dependence, but it should be noted that this method may lead to the underestimation of the real value of slope coefficient. An additional and the most powerful source of error in the radon epidemiological studies is the influence of smoking and the correlation between smoking status and radon concentration in dwellings. The modeling results demonstrated that maximum attention should be paid to the stratification by smoking status and other possible factors simultaneously influencing radon concentration in dwellings and lung cancer incidence.
\end{abstract}

Key words: radon, case-control study, errors influence, regression calibration, SIMEX.

DOI: $10.21175 /$ RadJ.2016.01.015

\section{INTRODUCTION}

Analysis of epidemiological data shows that the results of epidemiological case-control studies considerably depend on the quality of initial data. The correct selection of control group is required to obtain a non-biased assessment of dependence of observed effect (in our case lung cancer incidence) on the influencing factor (radon exposure). The stratification of case and control groups by sex, age and smoking status are typically used at most case-control radon studies [1-4]. It should be noted that, even at completely correct stratification of case and control groups by sex, age, smoking and other factors, the resulted exposure-effect dependence is affected by the influence of measurement errors or errors in assigning the results of current measurements to exposure interval $5-30$ years prior to the time of risk assessment. These errors inevitably have an impact on the results of the evaluation of the coefficients, describing the "exposure-effect" dependence. Therefore, before the beginning of epidemiological studies, it seems appropriate to conduct a preliminary analysis of the effect of errors on the results of planned studies. An example of such work is the paper [5], published before the start of large-scale studies on the radon epidemiology in dwellings in North America. Analysis of the effect of errors in the evaluation of exposure on the results of epidemiological studies has been made. It is assumed that, in the initiation of lung cancer, biologically significant period of exposure to radon is an exposure period of 25 years, ranging from 5 to 30 years before the risk assessment. It was stated that it was important to make a distinction between the measurement errors and errors in the assessment of individual exposure. In paper [5], it is noted that the published data on case-control studies have substantial heterogeneity and do not allow an unambiguous conclusion about the relationship between exposure to radon in dwellings and lung cancer. It was concluded that, due to errors in the measurement of radon concentrations and the estimation of radon exposures, a single study cannot provide unambiguous lung cancer risk estimates. It was recommended to include procedures to estimate the distribution of exposure errors in the design of indoor radon studies, and analyses of studies should be adjusted for exposure errors.

At present, three combined studies on radon in dwellings and its influence on the incidence of lung cancer are completed in the world. They include a combined analysis of 7 North American case-control studies [4], 13 studies in Europe [1] and 2 studies in China [3].

The latest study (China) was excluded from further consideration due to reasonable doubts about the quality of the measurements of radon concentration. In particular, there were high concentrations of radon-

\footnotetext{
* The paper was presented at the Third International Conference on Radiation and Applications in Various Fields of Research (RAD 2015), Budva, Montenegro, 2015.

${ }^{* *}$ Contact: onischenko@ecko.uran.ru
} 
220 (thoron) that influenced the assessment of human exposure to radon-222 [9] in some premises.

In a combined analysis of seven North American studies [4], the pooled size of the sample was 3662 cases and 4966 controls. In the analysis, it was found that the odds ratio corresponded to a value $11 \%$ per 100 $\mathrm{Bq} / \mathrm{m}^{3}$ (CI o $-28 \%$ ). Later, to improve the accuracy of the estimates, only the studies in which people lived in no more than two homes, with the instrumental evaluation of radon exposure spanning over a period of more than 25 years were included in the analysis. As a result, the sample was reduced to 1910 cases and 2651 controls, and the slope increased to $18 \%$ (CI $2-43 \%$ ). No further analysis of the influence of measurement errors or errors in the exposure assessment was conducted. In fact, the resulting dependence of exposure-effect was obtained on the basis of observed data, although quite reliable (so called naïve evaluation).

The most detailed case-control study is a combination of 13 European studies [1] with the total number of cases of lung cancer 7148 and controls 14208. The combined study included the results of studies conducted in 9 countries. As a result, the risk assessment of lung cancer from the measured radon concentration was $8.4 \%$ per $100 \mathrm{~Bq} / \mathrm{m}^{3}$ (CI $3-15.8 \%$ ). Darby et al. [1] noted that the influence of radon exposure errors leads to an underestimation of the exposure-effect dependence. In this regard, the correction of the measured values of radon concentration to obtain the most probable values of the true concentration of radon was performed. The detailed approach to the correction of the influence of radon measurement errors is presented in work (10).

Until now, Russia has not conducted large-scale epidemiological studies on the effect of radon exposure on lung cancer. Only few small pilot studies were performed in two cities of the Middle Urals and Lermontov city on Caucasus [6-8]. A large-scale study on the influence of radon on the incidence of lung cancer in Russia is at the planning stage now. The purpose of this planned study is to obtain the reliable data on radon exposure-effect dependence in a single study, conducted with a uniform approach to conducting the measurements and data analyzing.

The aim of this work is to model such research, to assess the impact of errors on the research results and to select methods that allow taking into account the impact of these errors. In our study, the approaches to the modeling of radon studies described in works [5, 10] were used.

\section{MATERIALS AND METHODS}

At the first stage of modeling, the distributions of radon concentrations in a given population were generated. Based on numerous data, the assumption that the distribution of radon concentrations was quite precisely described by lognormal distribution was made. Therefore, in the simulation, the values of the geometric mean (GM) and the standard deviation of the logarithms of radon concentration $\left(\sigma_{\mathrm{LN}}\right)$ were set.
Lung cancer incidence was modeled in the following way:

- The baseline value of spontaneous lung cancer incidence was chosen. When using the age-specific incidence $\lambda_{o}(t)$, it was assumed that the frequency of spontaneous occurrence of lung cancer for nonsmoking men and women was, practically, the same [12]. The data were approximated by fitting the function smoothly, depending on age $t$ at the time of the risk assessment.

- The age distribution of the population for $t>40$ years was modeled by decreasing function generally corresponding to the probability of survival to a certain age. Ages up to 40 years have not been considered due to low probability of spontaneous occurrence of lung cancer.

- For each member of the population, smoking status was modeled at random, and then the agespecific probability of spontaneous occurrence of lung cancer has been multiplied by the coefficient of relative risk for a group of smokers. It was assumed that $60 \%$ of males and $30 \%$ of females have been exposed to smoking (smokers of different intensity or ex-smokers). The types of smoking groups and the relative risk values have been chosen in accordance with [1].

- The probability of lung cancer occurrence as a result of the combined action of radon and smoking was calculated as:

$$
P(t, \text { smoking }, R n)=\lambda_{0}(t) \cdot R R_{s m}\left(1+C_{R n} \cdot R R_{R n}\right),(1)
$$

where $R R_{s m}$ - the relative risk of lung cancer due to smoking in selected smoker's group; $R R_{R n}$ - the relative risk of lung cancer due to radon exposure (o.16 per $100 \mathrm{~Bq} / \mathrm{m}^{3(1)}$ ); $C_{R n}$-average radon concentration influencing a given individual.

- Using a random numbers generator, the number uniformly distributed in the interval 0 to 1 was generated. It was considered that lung cancer was realized and this individual belonged to the group "case" under the condition:

$$
R N D \leq P \text { (t, smoking, } R n \text { ). }
$$

Otherwise, a person belonged to the group "control". For given values of the geometric mean of radon concentration and the standard deviation $\sigma_{\mathrm{LN}}, 10^{6}$ stories were generated. The same numbers of men and women have been modeled. The modeled data on lung cancer incidence were in good agreement with European statistical data [11].

To estimate the influence of errors, it has been assumed that the dominant type of errors are multiplicative errors, which are described by a lognormal distribution law $[5,10]$. The value of the multiplicative error is, due to errors in the calibration of detectors, the influence of seasonal variations of radon, uncertainties associated with the transition from the current values of radon concentration to the weighted average over a long period, etc., constant over the entire range of radon concentration in a given sample. To obtain the distribution of the values of radon concentration affected by the error influence, the 
individual "measured" radon concentrations were generated as

$$
C_{R n}^{\text {meas }}=\exp \left[\ln \left(C_{R n}^{\text {real }}\right)+\operatorname{NORM} \cdot \operatorname{INV}\left(R N D ; 0 ; \sigma_{\text {err }}\right)\right](3)
$$

where NORM.INV - function which returns the inverse of the normal distribution for the specified mean and the standard deviation.

Separately, the effect of the error caused by a Poisson error associated with a random dispersion of the number of tracks registered by track detector was analyzed. This error is more strongly expressed in cases when the radon concentration is low, duration of exposure of the detector is short and the number of tracks registered by track detector is relatively small in comparison with the background track density. For significant concentrations of radon or prolonged exposure to radon, impact of this uncertainty is likely to be low. Nevertheless, the assessment of the impact of the error having differential influence in the range of measurements is necessary.

For modeling purposes, NRPB and RadoSys detectors were chosen for detection of alpha particles by CR-39 material and an optical system of tracks counting. These detectors have a sensitivity to radon $\varepsilon=2.1\left(\right.$ track $\left.\cdot \mathrm{cm}^{-2}\right) /\left(\mathrm{kBq} \cdot \mathrm{m}^{-3} \cdot \mathrm{h}\right)$ with a background

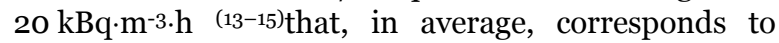
track density $N_{b g r}=41$ track $\cdot \mathrm{cm}^{-2}$. Calculation of the relative Poisson error for the track detector was carried out as:

$$
\sigma_{\text {count }}=\frac{\sqrt{C_{R n}^{r e a l} \cdot T_{e x p} \cdot \varepsilon+N_{b g r}}}{C_{R n}^{r e a l} \cdot T_{\text {exp }} \cdot \varepsilon-N_{b g r}}
$$

where $T_{\exp }-$ duration of detector exposure. The influence of Poisson errors which depended on individual radon concentration was taken into account in correspondence with the approach used for other kind of errors (Equation 3).

In the calculation of odds ratios (OR) for different ranges of exposures to radon, several approaches to select a range of calculations were used:

- approximately the same number of members of the case group in each of the intervals;

- approximately the same total number of members of the case and control groups in each of the intervals;

- manual settings of intervals.

Odds ratios for different options have been calculated using a specialized program designed in the Institute of Industrial Ecology. An error estimation is not a trivial task for the slope of the dose-effect in situations where the odds ratio in each of the sub range has its own asymmetrical confidence interval. To solve it, a numerical method of generating Monte Carlo sets of OR for all intervals, taking into account their uncertainties and distribution law has been used. For each randomly generated set of odds ratio, the slopes of exposure-effect dependence were calculated and the overall confidence intervals for the slope were estimated.

\section{RESULTS AND DISCUSSION}

\subsection{The influence of the selection of the control group on exposure-effect dependence}

As it is well known, incorrectly selected control group at epidemiological studies brings the greatest mistake in the interpretation of the results of the analysis. For an assessment of the influence of this factor at imitating modeling, four various options of selection of control groups were considered:

- control group corresponds to the average distribution by sex, age and smoking status of the general population exposed to radon (population control);

- control group is aligned with the case group by the ratio of men and women; other factors (age and smoking) are not standardized and correspond to the distribution of the general population (standardization by sex);

- control group is aligned with the case group by sex and age; smoking status is not aligned (standardization by age and sex);

- $\quad$ for each member of the case group, a pair matched by sex, age and smoking status is selected (copypair standardization).

At the first stage of modeling, it was believed that radon levels affecting the population in each of the samples did not correlate with factors such as sex, age and smoking status. In general, it is not fully consistent with the experimentally observed facts, because lower average radon concentration in the dwellings of smokers was noted in various sources $[16,17]$. This fact may have an explanation both in changing the regime of the dwellings (perhaps, more frequent ventilation), and in different socio-economic status of smokers and nonsmokers (it is known that the percentage of smokers is higher in groups with low socio-economic status), which may affect the quality of housing.

Eight model groups of population with $10^{6}$ people in each were generated. The GM values of radon concentration were set in the range from 20 to 100 $\mathrm{Bq} / \mathrm{m}^{3}$, the $\sigma_{\mathrm{LN}}-$ in the range from 0.8 to 1.2 .In each group $~ 800$ lung cancer cases among the males and $\sim 200$ cases among females were observed. The difference was explained by a different smoking level for females and a smaller level of spontaneous lung cancer rate. The number of cases of lung cancer obtained by modeling for different samples is shown in Fig.1. Later, for all modeled "cases", different "controls" were selected than for the other "population". During the modeling, the ratio between the number of members of case and control groups always was $1: 2$. 


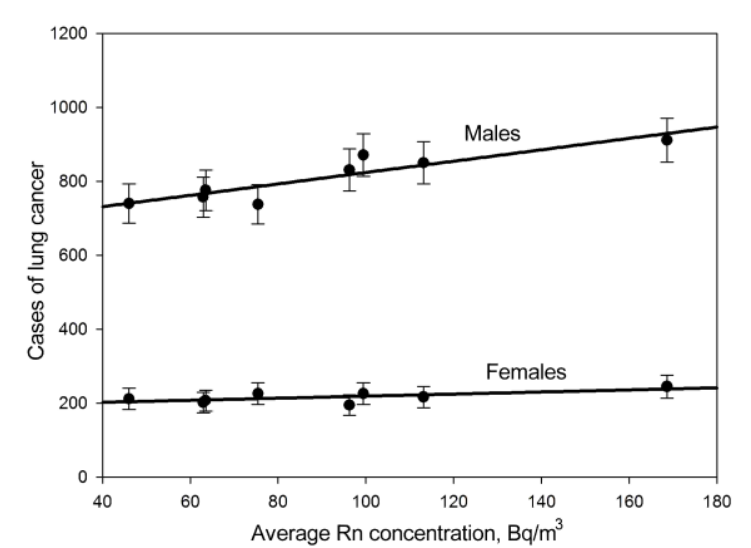

Figure 1. The number of cases of lung cancer obtained by modeling for different samples in dependence on average radon concentration (no correlation between smoking and radon concentration)

The number of expected cases of lung cancer for males in dependence on radon concentration is described with statistical significance by linear regression (standard error shown):

$$
N_{m}=(670 \pm 28)+(0.0014 \pm 0.0003) \cdot C_{R n}^{r e a l}
$$

In fact, in the modeling of a cohort study, we can observe that the slope of the lung cancer incidence in dependence on the average radon levels, in general, corresponds to the originally specified level. Because of the smaller number of cases observed, slope dependence for females was $0.0035 \pm 0.0016 \mathrm{~Bq}^{-1}$ and was not statistically significant.

The analysis of OR dependence on radon concentration demonstrated a set of features:

- Even in the studies with 800 - 900 cases and $1600-1800$ controls, there is significant influence of random character of radon concentration distribution on lung cancer incidence. The OR value dependences on the "real" radon concentrations were in the range from 0.09 to 0.27 per $100 \mathrm{~Bq} / \mathrm{m}^{3}$ (at the given value 0.16 )in different samples.

- In individual samples, the value of exposure-effect slope can be unpredictably changed $\sim 1.5-2$ times in dependence on the number of intervals used for calculation of $\mathrm{OR}$ values.

- $\quad$ For a combined group containing 8204 cases and 16408 controls, the value of exposure-effect slope is in the range from 0.13 to 0.17 per $100 \mathrm{~Bq} / \mathrm{m}^{3}$ (average value $0.16 \pm 0.01$ ). This value corresponds to initially established value 0.16. Even for such big case and control groups, dependence of exposure-effect slope on the number of intervals used for calculation of OR values can be observed.

- For the slope of the odds ratios dependence on the radon concentration, there are no statistically significant differences between the calculations performed for different methods of stratification of the control group: population control, control by sex, control by sex and age, and the control copy-pair.

The most important result obtained during the analysis of the groups with no correlation of radon concentration with other factors (such as sex, age or smoking status) is the absence of dependence of exposure-effect slope coefficient on the type of control group selection. In fact, this means, that in the absence of a correlation between the concentration of radon in dwellings and the factors influencing the incidence of lung cancer (sex, age, smoking), we cannot watch the changes in the slope of the function exposure-effect with any method of selection of the control group. This can be explained by the fact that the radon concentration distribution in the control group is identical to the one in any group obtained at random from the general population.

So, in epidemiological study, it is very essential to find and take into account the confounding factors influencing both lung cancer incidence and radon concentration in dwellings. As it was mentioned above, the only factor simultaneously influencing both lung cancer incidence and radon concentration is smoking. According to the data [16], the average radon concentration was approximately $10 \%$ smaller in the smoker's dwellings than in the dwellings of nonsmokers. For other influencing factors, such as sex, age etc. there are no data on their influence on average radon concentration in dwellings.

To take into account the effect of smoking on the average concentration of radon in the dwellings, the scheme of the model experiment was modified. If the considered individual had a smoking status other than "nonsmoker", the radon concentration in the individual's dwelling was reduced in value randomly and uniformly distributed in the range $(0-20) \%$ from the initial concentration. Like in the previous modeling, 8 groups in $10^{6}$ individuals with the same values of GM of radon concentration were generated. The number of cases of lung cancer, obtained by modeling for different samples is shown in Fig. 2.

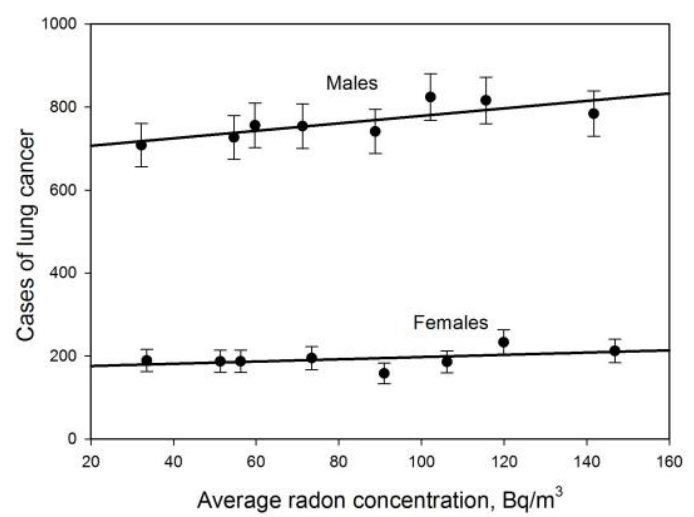

Figure 2. The number of cases of lung cancer, obtained by modeling for different samples, in dependence on average radon concentration (correlation $-10 \%$ between smoking and radon concentration)

The number of expected cases of lung cancer for males, like in the previous case, is significantly described by regression (standard error is shown):

$$
N_{m}=(689 \pm 26)+(0.0011 \pm 0.0004) \cdot C_{R n}^{r e a l}
$$

For females, the observed slope of the dependence on the average radon concentration was $0.0028 \pm$ $0.0021 \mathrm{~Bq}^{-1}$ and was not statistically significant. In 
modeling of the cohort study, it can be observed that in the case of negative correlation between smoking status and radon concentration, the slope of exposureeffect dependence is less than in case of absence of correlation.

In the case-control analysis of the combined sample, it was found that the slope exposure-effect essentially depends on the selection of the control group. The improvement of the selection of the control group increases the growth of lung cancer incidence per unit of radon concentration (Table 1).

Table 1.Odds ratio dependence on the "real" radon concentrations for different kinds of selection of control group

\begin{tabular}{|l|c|}
\hline Selection of control group & $\begin{array}{l}\text { Slope coefficient per } \\
100 \mathrm{~Bq}^{-1}(95 \% \mathrm{CI})\end{array}$ \\
\hline Population control & $0.038 \pm 0.014$ \\
Standardization by sex & $0.079 \pm 0.020$ \\
Standardization by age and sex & $0.070 \pm 0.010$ \\
Standardization copy-pair & $0.120 \pm 0.012$ \\
\hline
\end{tabular}

Like in the modeling of the cohort study, it is possible to observe that the slope of exposure-effect dependence is smaller than initially used in modeling risk coefficient 0.16 per $100 \mathrm{~Bq}^{-1}$. The decrease of the slope of exposure-effect on the negative correlation between smoking status and indoor radon concentration can be explained by the change of smoker/nonsmoker ratio in different ranges of OR calculation. At the absence of correlation between smoking status and indoor radon concentration, the smoker/nonsmoker ratio is practically the same for all the ranges of radon concentration (Fig. 3a). At such correlation, the statistically significant increase of nonsmoker part at high radon concentration can be observed (Fig. 3b). Because of the fact that the risk of lung cancer incidence for nonsmokers is considerably less than for smokers, the OR values at high radon concentrations are decreased. It leads to a total decrease of the slope of exposure-effect.
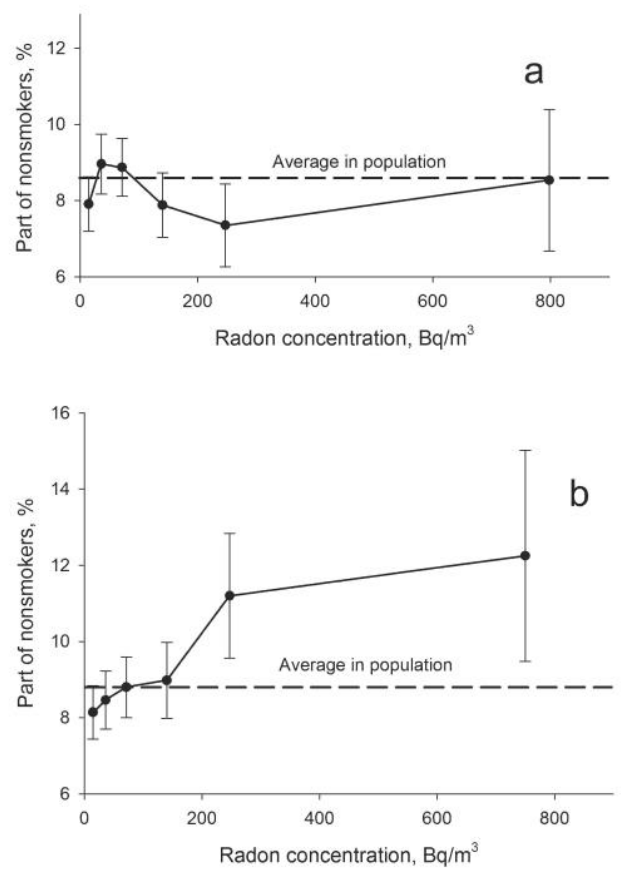

Figure 3. Dependence of the part of nonsmokers on indoor radon concentration: a - no correlation between smoking and radon concentration, $\mathrm{b}$ - correlation $-10 \%$ between smoking and radon concentration

3.2. The influence of radon exposure errors on exposure-effect dependence and the correction of this influence

For the assessment of radon exposure errors on the exposure-effect dependence, the multiplicative error with $\sigma_{\text {err }}=0.50$ was assumed. The additional influence of Poisson errors led to increasing this value for the combined sample to $\sigma_{\text {err }}=0.55$.

For the general sample, without the correlation between smoking and radon concentration, the influence of errors decreased the slope of exposureeffect dependence from $0.16 \pm 0.01$ to $0.11 \pm 0.01$ per $100 \mathrm{~Bq}^{-1}(95 \% \mathrm{CI})$.

For the sample with negative correlation between smoking and radon concentration, the slope of exposure-effect dependence (for copy-pair standardization) decreased from $0.120 \pm 0.012$ to $0.077 \pm 0.015$ (95\% CI). It demonstrates that "naïve" assessments based on observed values of radon concentrations can significantly underestimate the exposure-effect dependence.

In analysis of epidemiological data, there are a number of techniques, which allows improving the influence of the errors. In our work, we used the method of regression calibration [10] and the so-called SIMEX method [18].

Method of regression calibration [10] is used to replace the unobservable true value of the variable in the regression by the expected from the obtained measured value. To estimate the expected "real" value $x$ from observed value $z$, the next expression was used:

$$
\mu_{x \mid \mathrm{z}}=\left(\frac{1}{\sigma_{\text {err }}^{2}}+\frac{1}{\sigma_{A}^{2}}\right)^{-1}\left(\frac{z}{\sigma_{\text {err }}^{2}}+\frac{\mu_{A}}{\sigma_{\text {err }}^{2}}\right)
$$

where $\sigma_{e r r}^{2}-$ error variation; $\sigma_{A}^{2}-$ observed variation of measured radon concentration values in control group corrected on the influence of $\sigma_{e r r}^{2} ; \mu_{A}-$ expected average value of radon concentration in the control group. For multiplicative errors the logarithmic values of concentrations and variances should be used. The use of method of regression calibration for assessing the "true" value of the slope of exposure-effect dependence for the sample with negative correlation between smoking and radon concentration and copypair standardization gives the value $0.13 \pm 0.04$ per 100 $\mathrm{Bq}^{-1}$ (95\% CI). This value completely corresponds to the assessment made by the use of initial "real" values of radon concentrations unaffected by errors.

SIMEX method is of particular interest [18]. The essence of the method is to add an extra error to the measured data obtained from the study and the evaluation of the impact of additionally introduced measurement errors on the results of the assessments made by the method of regression. Suppose we have a "naive" estimate of slope coefficient $\beta$ and an additional set of data with consistent increase in error variances $(1+\lambda) \cdot \sigma_{A}^{2}$. During the analysis, the dependence of $\beta$ value on the parameter $\lambda$ is plotted. 
The required value $\beta_{\text {true }}$ can be obtained by extrapolating the observed dependence $\beta$ on $\lambda$ to the value $\lambda=-1$. For multiplicative errors, logarithmic coordinates should be used (logarithmic dispersion) (Fig. 4). In this method, it is necessary to consider that additional modeling of the random error should be performed repeatedly to obtain statistically significant evaluation of the expected value.

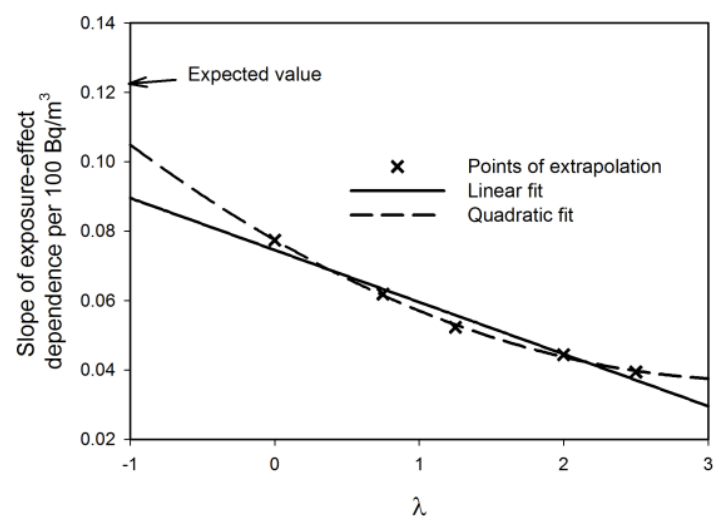

Figure 4. The example of the use of SIMEX method for assessing of the real value of the exposure-effect slope

In general, the SIMEX method also allows obtaining the good assessment of expected value of the slope of exposure-effect dependence. The quadratic or cubic extrapolations give better results than linear extrapolation but it should be noted that SIMEX method leads to the underestimation of the real value of the slope coefficient.

\section{ConClusions}

The analysis allows making the following conclusions. When planning a large-scale national or international epidemiological study to detect the lung cancer incidence due to exposure to radon in the dwellings, the researchers should focus on the following issues:

- Thorough identification of factors affecting both the concentration of radon in homes and lung cancer. Identify, if possible, the numerical values of the coefficients of proportionality for these factors.

- Comprehensive assessment of the factors influencing the error of radon exposure assessment and numerical evaluation of all errors.

- The analysis of the possible impact of errors on the observed values of the exposure-effect dependence and the choice of optimal techniques to obtain undistorted value of the desired parameter.

- Results of exposure-effect dependence essentially associated with the choice of intervals in which the odds ratios are calculated. In this regard, the critical rule for choosing such intervals should be worked out.

- Decrease in the slope of the exposure-effect dependence at presence of negative correlation between smoking and radon should be taken into account. It should be noted that this effect was observed when the "real" values of radon concentration were used. The influence of such factors on the results of the studies should be examined very carefully.

- The modeling demonstrated that to obtain an acceptable statistical accuracy $(\sim 15 \%$ at $95 \% \mathrm{CI})$, the planned study should be comparable in scale to the combined European radon study, i.e. the number of cases should be within $6000-8000$ and the number of controls $12000-16000$. In planning such study, the quality of selection of the control group should initially be at a high possible level. The detailed analysis of the numerical values of different errors influencing the radon exposure assessment are also very important due to the fact that error values will inevitably be used for the correction of naïve evaluations of exposure-effect dependence.

Acknowledgement: This work was supported by the Ural Branch of Russian Academy of Science (project 15-3-2-10).

\section{REFERENCES}

1. S. Darby et al., "Residential Radon and Lung Cancer: Detailed Results of a Collaborative Analysis of Individual Data on 7148 Subjects with Lung Cancer and 14208 Subjects without Lung Cancer from 13 Epidemiologic Studies in Europe," Scand. J. Work. Environ. Health, vol. 32, suppl. 1, pp. 1-83, 2006.

2. S.X. Yao et al., "Exposure to Radon Progeny, Tobacco use and Lung Cancer in a Case-Control Study in Southern China," Radiat. Res., vol. 138, no. 3, pp. 326336, 1994.

3. J. H. Lubin et al. "Risk of Lung Cancer and Residential Radon in China: Pooled Results of two Studies", Int. J. Cancer, vol. 109, no. 1, pp. 132-137, Mar. 2004.

4. D. Krewski et al., "Residential Radon and Risk of Lung Cancer: a Combined Analysis of 7 North American CaseControl Studies," Epidemiology, vol. 16, no. 2, pp.137145, Mar. 2005.

5. J.H. Lubin, J.D. Boice Jr. and J.M. Samet, "Errors in Exposure Assessment, Statistical Power and the Interpretation of Residential Radon Studies," Radiat. Res., vol. 144, pp. 329-341, Dec. 1995.

6. Е.В. Ползик, В.Л. Лежнин и В.С. Казанцев, "К проблеме оценки влияния радона на развитие рака легких," Радиационн. биол. Радиоэк., т. 44, № 2, с. 207-215, 2004. (E.V. Polzik, V.L. Lezhnin and V.S. Kazantsev, "Towards the Issue of Evaluating the Radon Effects on Lung Cancer," Radiat. Biol. Radioecol., vol. 44, no. 2, pp. 207-215, 2004.)

7. V.L. Lezhnin, E.V. Polzik, V.S. Kazantsev, M.V. Zhukovsky and O.A. Pakholkina, "A Multifactorial Assessment of Carcinogenic Risks of Radon for the Population Residing in a Russian Radon Hazard Zone," Arch. Oncol., vol. 19, no. 1-2, pp. 3-8, 2011.

8. M. Zhukovsky, A. Varaksin and O. Pakholkina "Statistical Analysis of Observational Study of the Influence of Radon and Other Risk Factors on Lung Cancer Incidence," Rad. Prot. Dosim., vol. 160, no. 1-3, pp. 108-111, 2014. 
9. Y. Yamada et al., "Radon-Thoron Discriminative Measurements in Gansu Province, China, and their Implication for Dose Estimates," J. Toxicol. Environ. Health, Pt. A, vol. 69, no.7, pp. 723-734, 2006.

10. T. Fearn, D.C. Hill and S.C. Darby, "Measurement Error in the Explanatory Variable of a Binary Regression: Regression Calibration and Integrated Conditional Likelihood in Studies of Residential Radon and Lung Cancer”, Dept. of Statist. Sci., Univ. College London, England, Research Rep. 280, Oct. 2007.

11. "European Detailed Mortality Database (DMDB)," World Health Organization, Regional Office for Europe. Retrieved from: http://data.euro.who.int/dmdb/; Retrieved on: 2014.

12. R. Peto, A.D. Lopez, J. Boreham, M. Thun and C. Heath Jr., "Mortality from Tobacco in Developed Countries: Indirect Estimation from National Vital Statistics," Lancet, vol. 339, pp. 1268-1278, May 1992.

13. C.B. Howarth, "Results of the 2006 Health Protection Agency Intercomparison of Passive Radon Detectors,"
Health Protection Agency, 1st ed., Chilton, UK, 2007, HPA-RPD-053.

14. C.B. Howarth, "Results of the 2007 Health Protection Agency Intercomparison of Passive Radon Detectors," Health Protection Agency, Chilton, UK, Nov. 2009, HPA-RPD-060

15. Z. Daraktchieva, C.B. Howarth and R. Algar "Results of the 2011 HPA Intercomparison of Passive Radon Detectors," Health Protection Agency, Chilton, UK, May 2012, HPA-CRCE-033.

16. B.S. Cohen, "Variation of Radon Levels in U.S. Homes Correlated with House Characteristics, Location, and Socioeconomic Factors," Health Phys., vol. 6o, no. 5, pp. 631-642, May 1991.

17. Z.S. Zunic et al., "Radon Survey in the High Natural Radiation Region of Niska Banja, Serbia," J. Environ. Radioact., vol. 92, no. 3, pp. 165-174, 2007.

18. J.R. Cook and L.A. Stefanski, "Simulation-Extrapolation Simulation in Parametric Measurement Error Models," J. Amer. Stat. Asso., vol. 89, no. 428, pp. 1314-1328, Dec. 1994. 